\title{
La demanda de gasolinas en México: Efectos y alternativas ante el cambio climático ${ }^{\mathrm{a}}$
}

\author{
Orlando Reyes, * Roberto Escalante** y Anna Matas***
}

\section{RESUMEN}

El autotransporte es de los sectores más contaminantes en México, generando alrededor de 17\% del total de emisiones de $\mathrm{CO}_{2}$. El consumo de gasolina y diesel son la principal fuente de estas emisiones. Este artículo analiza empíricamente la demanda de gasolinas del sector automotor en México durante el periodo 1960-2008. Las estimaciones de las elasticidades de largo y corto plazos del precio e ingreso fueron: $-0.285,-0.041,1.004$ y 0.721 , lo que implica que la demanda de gasolinas es sensible a la trayectoria del ingreso e inelástica a los precios. Por tanto, un crecimiento económico continuo, sin una adecuada política de precios, generará un aumento en el consumo de gasolinas. Esta situación puede ser más grave al considerar los efectos del cambio climático suponiendo una demanda relativamente constante. Bajo estas circunstancias es necesario implantar diversas políticas públicas simultáneamente para frenar las consecuencias del consumo de gasolinas sobre el cambio climático.

Palabras clave: demanda de gasolinas, elasticidades ingreso y precio, sector autotransporte, políticas económicas, emisiones de bióxido de carbono y técnicas de cointegración.

Clasificación JEL: R41, O13, C32.

\begin{abstract}
The transportation sector is one of the most polluting sectors in Mexico, generating around $17 \%$ of total $\mathrm{CO}_{2}$ emissions. Moreover, the consumption of gasoline and diesel are the main source of these emissions. This document empirically analyses the fuel demand in the transportation sector of Mexico over the period 1960-2008. The results show that the long -and short- run price and income elasticities in fuel consumption are: $-0.285,-0.041,1.004$ and 0.721 . This implies that fuel demand is sensitive to the trajectory of income and is inelastic to prices. Therefore, a continuous economic growth without a suitable price policy will generate an increase in gasoline consumption. This situation could be worst if we take into consideration the effects on climate change. Under these circumstances it is necessary to implement simultaneously a variety of public policies to control the impact of fuel consumption on climate change.
\end{abstract}

Key words: gasoline demand, income and price elasticity, road transport, economic policy, carbon dioxide emissions and cointegration techniques.

Classification JEL: R41, O13, C32.

\footnotetext{
${ }^{a}$ Los autores agradecen los valiosos comentarios de Horacio Catalán y Luis M. Galindo. Se aplica desde luego el descargo usual de los errores. Este trabajo se realizó con el apoyo financiero del Conacyt y del MAEC-AECID. * Doctorante en economía aplicada en la Universidad Autónoma de Barcelona, España: orlando.reyes@campus.uab.cat.** Doctor en desarrollo rural por la Universidad de Londres (Wye College): semerena@servidor.unam.mx. *** Doctora en economía aplicada por la Universidad Autónoma de Barcelona, España: anna.matas@uab.cat.
} 


\section{INTRODUCCIÓN}

El transporte es una de las actividades más importantes dentro del sistema económico mexicano. Sin duda, es una fuente indispensable para el funcionamiento del aparato productivo. De acuerdo con el Programa Sectorial de Comunicaciones y Transporte (2007-2012), en los pasados 10 años creció a una tasa anual promedio de $6.6 \%$, lo que representa más del doble de lo que creció la economía mexicana en su conjunto durante el mismo periodo. En 2007, su participación en el producto interno bruto (PIB) representó cerca de 14\%. Asimismo, aporta directamente 2.2 millones de empleos, lo que representa $5.1 \%$ del mercado laboral.

El sector del transporte también es una de las fuentes más importantes de emisiones de gases de efecto invernadero (GEI). De acuerdo con cifras del Instituto Nacional de Ecología (INE) (2006), se estima que en 2002 México generó el equivalente a 643183 gigagramos $(\mathrm{Gg})^{1}$ en unidades equivalentes $(\mathrm{UE})^{2}$ de bióxido de carbono $\left(\mathrm{CO}_{2}\right)$, volumen que lo sitúa dentro de los 15 principales países emisores, con una contribución aproximada de $1.5 \%$ de las emisiones globales. En lo que respecta a las fuentes responsables de emisiones, la mayor contribución corresponde al sector energético con $61 \%$, de las cuales, respecto a la quema de combustibles fósiles en fuentes móviles (sector transporte), equivalen a 29\%. Cabe destacar que dentro de este sector el transporte por carretera es el principal responsable de las emisiones de $\mathrm{CO}_{2}$, con poco más de 90 por ciento. ${ }^{3}$

La dependencia del petróleo como fuente de energía para el transporte produce condiciones cada vez más desfavorables en los ámbitos social, político y ambiental (Higgins y Higgins, 2005). En este contexto, en la actualidad el sector autotransporte es uno de los mayores impulsores de emisiones de $\mathrm{CO}_{2}$. Dados los objetivos fijados para la reducción de las emisiones, es necesario conocer el

\footnotetext{
${ }^{1} \mathrm{El} \mathrm{Gg}$ es la unidad de medida de masa equivalente a $10^{9}$ gramos, empleada para las emisiones de GEI. Un Gg equivale a 1000 toneladas (INE, 2006).

${ }^{2}$ Concentración de $\mathrm{CO}_{2}$ que podría causar el mismo grado de forzamiento radiactivo que una mezcla determinada de GEI. Medida que se utiliza para comparar las emisiones de distintos GEI con base en su potencial de calentamiento global (INE, 2006).

${ }^{3}$ En España el sector automotor aporta aproximadamente 25\% del total de emisiones de $\mathrm{CO}_{2}$ (Ministerio de Medio Ambiente y Medio Rural y Marino, MARM, 2010). Para el caso de los Estados Unidos, de acuerdo con la Agencia de Protección del Medio (EPA), aproximadamente 20\% del total de las emisiones de $\mathrm{CO}_{2}$ que se generan provienen de los vehículos de pasajeros (automóviles y camiones ligeros). Estimaciones de Canadá y el Reino Unido muestran que 25\% de las emisiones de $\mathrm{CO}_{2}$ son provocadas por el sector del transporte (Basso y Oum, 2007). En México, el sector autotransporte contribuyó aproximadamente con $17 \%$ de la cantidad total de emisiones de $\mathrm{CO}_{2}$ (INE, 2006).
} 
impacto de la quema de combustibles fósiles en dichas emisiones para dimensionar correctamente las decisiones de política de transporte orientadas a disminuir el consumo energético.

Este documento estima la demanda de gasolinas en México y analiza las relaciones entre el consumo de gasolinas en el sector automotor y la generación de $\mathrm{CO}_{2} \cdot{ }^{4}$ Asimismo, realiza diversas proyecciones de la demanda de gasolinas para los próximos años, considerando un escenario base y tres escenarios alternativos. La evidencia disponible indica que existe una fuerte dependencia de la trayectoria del consumo de combustibles respecto al comportamiento del ingreso y que es poco sensible a los precios. En los próximos años una mejora del crecimiento económico en México, sin una adecuada política de precios, conducirá a un aumento paulatino de la demanda de gasolina, con consecuencias negativas sobre el ambiente. El trabajo concluye que es necesario que se implanten diversas medidas para buscar separar el crecimiento económico del consumo de gasolinas y de las emisiones de $\mathrm{CO}_{2}$ asociadas.

La investigación está estructurada en tres secciones. En la sección I se hace una revisión de estudios previos de la demanda de gasolinas. En la sección II se muestra la especificación de la demanda de gasolinas y se discuten las técnicas econométricas que se emplearon. La descripción de los datos y los resultados del modelo se presentan en la sección III. En ese mismo apartado se muestran los ejercicios de proyección sobre la sensibilidad de la demanda ante cambios en las variables explicativas y se presenta un análisis sobre la relación de la demanda de gasolinas y las emisiones de $\mathrm{CO}_{2}$. $\mathrm{Al}$ final se exponen las conclusiones y otras consideraciones.

\section{REVISIÓN DE ESTUDIOS PREVIOS}

El interés por la demanda de gasolinas cobró especial relevancia después de las crisis del petróleo en los años setenta. Recientemente, las fluctuaciones en el precio mundial del petróleo y la preocupación por las emisiones de GEI han dado lugar a un renovado interés en la estimación de los factores determinantes de la demanda de gasolinas (Nicol, 2003). Existen estudios recopilatorios que analizan las principales características (variables, formas funcionales, métodos de estimación que incluyen especificaciones de sección cruzada, de series de tiempo y de datos panel, con estructuras de rezagos, con microdatos y datos agregados) de

\footnotetext{
${ }^{4}$ Los términos consumo y demanda se utilizarán indistintamente en este documento.
} 


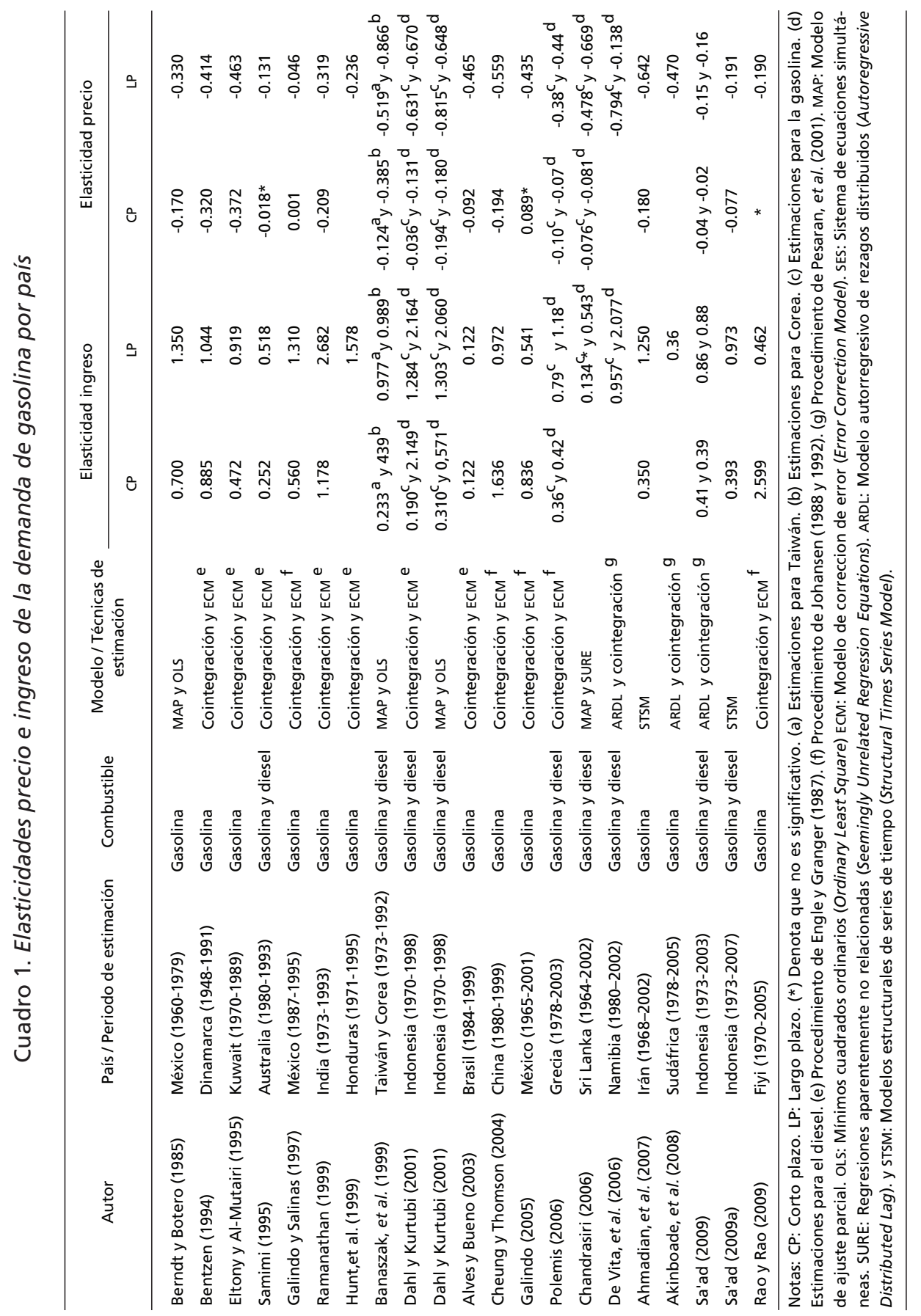


la demanda de gasolinas (Drollas, 1984; Dahl, 1986; Oum, 1989; Dahl y Sterner, 1991a y 1991b; Goodwin, 1992; Sterner y Dahl, 1992; Espey, 1998; Graham y Glaister, 2002 y 2004; Goodwin, et al., 2004; y Basso y Oum, 2007). Estos trabajos muestran que el consumo de gasolinas es influido negativamente por los precios, aunque éste es inelástico principalmente en el corto plazo, y se ve afectado positivamente por los ingresos, con elasticidades mayores a la unidad.

Una revisión de los estudios dedicados a la estimación de la demanda de gasolinas permite constatar que el uso de datos de series temporales es uno de los más habituales. En los años recientes estos estudios han aplicado técnicas econométricas que permiten modelar la naturaleza no estacionaria de las variables involucradas en la ecuación de manera explícita. Como resultado de la aplicación de técnicas de cointegración, los valores estimados para la elasticidad precio en el largo plazo son inferiores a los hallados en otros estudios, sin que por el momento exista una única explicación para este resultado.

El cuadro 1 ofrece una extensa revisión de los resultados de estudios que estiman las elasticidades ingreso y precio para la demanda de gasolinas. A grandes rasgos, se observa que la elasticidad precio de corto plazo es inferior a -0.4 , mientras que en el largo plazo la sensibilidad de la demanda es siempre superior y en algunos estudios alcanza valores próximos a -0.9.

Estos resultados coinciden con los presentados por Graham y Glaister (2002 y 2004) y Goodwin, et al. (2004), que a partir de una extensa revisión de estudios concluyen que los valores para la elasticidad precio a corto y largo plazos se sitúan alrededor de los rangos de -0.2 a -0.3 y -0.6 a -0.8 , respectivamente. Asimismo, la elasticidad ingreso de corto y largo plazos de la demanda de gasolinas para la mayor parte de los resultados de los estudios se encuentra en los intervalos de 0.35 a 0.88 y 0.86 a 1.6 , respectivamente. Es cierto, no obstante, que se observa una cierta variabilidad en los resultados, en parte debida a los distintos métodos de estimación utilizados y los datos empleados. Por tanto, es preciso establecer claramente el contexto en el que se llevan a cabo las estimaciones para una correcta interpretación de los resultados.

Para el caso mexicano, ${ }^{5}$ Berndt y Botero (1985) estiman la demanda de gasolinas para el sector transporte en el periodo 1960-1979. Las elasticidades

\footnotetext{
${ }^{5}$ También existen estudios que estiman la demanda de gasolinas con la metodología econométrica de datos panel. Así, por ejemplo, Berndt y Botero (1985) estiman un panel de datos dinámico con datos agregados a 14 regiones del país para el periodo 1973-1978. Eskeland y Feyzioglu (1997) estiman la demanda de gasolina para las 32 entidades federativas de México (1982-1988) a partir del método de momentos generalizado en primeras diferencias (PD-MMG). Haro e Ibarrola (2000)
} 
de corto y largo plazos del ingreso y los precios que obtuvieron fueron: 0.70, $1.35,-0.17$ y -0.33 . Los autores señalan que mientras las estimaciones resultan plausibles, su interpretación es algo ambigua para el análisis económico debido a la estructura simple de la especificación.

Asimismo, Galindo y Salinas (1997) analizan las condiciones de exogeneidad de la demanda de gasolinas en la Zona Metropolitana del Valle de México. No obstante que se estima una elasticidad precio relativamente pequeña, los autores sugieren que es posible utilizar el precio de las gasolinas como instrumento de política económica para regular el consumo de este producto. Por último, Galindo (2005) estima las demandas de los diferentes tipos energía. Los resultados indican que en México la demanda de energía es impulsada fundamentalmente por los ingresos y que la respuesta inelástica de los precios revela la necesidad de instrumentar medidas más drásticas para separar el consumo de energía y el crecimiento económico.

Finalmente, los resultados para el caso mexicano son muy diversos a consecuencia de las especificaciones y de los distintos métodos de estimación (Berndt y Botero, 1985; Galindo y Salinas, 1997; Eskeland y Feyzioglu, 1997; Haro e Ibarrola, 2000 y Galindo, 2005). Esto tiene consecuencias importantes. En primer lugar, los datos utilizados cubren cierto periodo de tiempo y ciertas características, lo que implicaría que con el tiempo las elasticidades podrían cambiar como consecuencia, por ejemplo, de cambios económicos, políticos y tecnológicos.

Esto dificultaría su uso para propósitos de política pública y de pronósticos. Asimismo, las técnicas econométricas han evolucionado y el tratamiento de los datos es más apropiado. Por tanto, las conclusiones que se han obtenido son un tema aún abierto.

\section{MODELO}

La revisión de los estudios permite señalar que la demanda de gasolinas se ha calculado con una variedad de enfoques y especificaciones. ${ }^{6}$ La mayoría de

estiman diferentes funciones de demanda para regiones fronterizas de México con los Estados Unidos, con el objetivo de analizar y determinar la sensibilidad de la demanda de gasolina comercializada en la zona fronteriza y estatal del norte de México durante el periodo 1995-1999.

${ }^{6} \mathrm{Al}$ igual que otros productos, la demanda de gasolina puede caracterizarse como la demanda de cualquier otro bien o servicio (Sterner, 2007), y en general se modela de manera similar a las demandas de energía (Masih y Masih, 1997; Bentzen y Engsted, 1999; y Galindo, 2005). 
variables comunes que se han incluido en las estimaciones contienen: ingresos, precios de las gasolinas, precios de otros combustibles, precios del transporte público y las características de los vehículos. ${ }^{7}$ Asimismo, en el análisis de la demanda de gasolina se debe distinguir entre el corto y el largo plazos, dado que en el largo plazo los individuos tienen mayor capacidad de respuesta. En efecto, la conducta de los consumidores varía de acuerdo con el periodo de tiempo en el que ajusta sus expectativas de consumo ante los cambios en el ingreso y en los precios.

Este estudio sigue el procedimiento de Johansen (1988 y 1992). Este enfoque permite estimar las distintas relaciones de cointegración que pueden existir entre la demanda de gasolinas, el PIB y los precios de la gasolina en la economía mexicana, por medio de modelos de vectores autorregresivos (VAR). El análisis econométrico de las variables de estudio con modelos VAR requiere identificar inicialmente el orden de integración. Segundo, si las series son no estacionarias, se evalúa si se cointegran.

Con ello se encuentra una relación de equilibrio en el largo plazo entre las variables y es posible obtener las elasticidades. Por último, de acuerdo con el teorema de representación de Granger (Engle y Granger, 1987), si en un conjunto de variables éstas se encuentran cointegradas entonces existe una representación de dichas variables en forma de ECM, y viceversa. En definitiva, si realmente hay cointegración entre las variables del modelo, la formulación inicial estática del mismo y su estimación toman relevancia y se presentan como una opción válida para tomar en cuenta, entre otras cosas porque estaría libre de correlaciones espurias.

La demanda total de las gasolinas $\left(g_{t}\right)$ para México se puede especificar como función del nivel de ingreso $\left(y_{\mathrm{t}}\right)$ y de los precios relativos de las gasolinas $\left(p_{\mathrm{t}}\right)$ :

$$
g_{\mathrm{t}}=\alpha+\beta y_{\mathrm{t}}+\gamma p_{\mathrm{t}}+\varepsilon_{\mathrm{t}}
$$

donde $\alpha$ es una constante, $\beta>0$ y $\gamma<0$ representan las elasticidades ingreso y precio de la gasolinas, respectivamente, y $\varepsilon_{\mathrm{t}}$ es el término de error que sigue los supuestos clásicos. Las letras en minúsculas simbolizan el logaritmo natural de las series.

\footnotetext{
${ }^{7}$ También existen especificaciones en las que la demanda de gasolina puede reflejar el número de kilómetros recorridos y la eficiencia energética de los vehículos utilizados (Baltagi y Griffin, 1983).
} 


\section{RESULTADOS Y PRONÓSTICOS}

\section{Datos}

Las series utilizadas corresponden a observaciones anuales del periodo de 1960 a 2008 en el ámbito nacional. Así, se consideró el consumo de gasolinas $\left(g_{t}\right)$ medido en millones de litros por año, agregando gasolina y diesel, y fue obtenido de la base de datos del anuario estadístico de Petróleos Mexicanos (Pemex). Como medida de ingreso se consideró el PIB $\left(y_{t}\right)$ a precios de 1993. Esta variable fue obtenida de las estadísticas históricas del Instituto Nacional de Estadística y Geografía de México (INEGI). También, se utilizaron de los precios relativos de la gasolinas $\left(p_{\mathrm{t}}\right)$, los cuales se obtuvieron considerando el índice general de los precios de las gasolinas respecto al índice nacional de precios al consumidor publicados por el Banco de México (Banxico). La evolución y sus componentes tendenciales ${ }^{8}$ del PIB, de la demanda de las gasolinas y los precios relativos de las gasolinas de la economía mexicana se presentan en la gráfica $1 .{ }^{9}$ Las series del PIB y consumo de gasolinas mantienen durante todo el periodo un comportamiento cíclico, fuertemente autocorrelacionado en torno de una tendencia ascendente (Blanchard, 1997). En tanto que los precios muestran cambios significativos en su tendencia hasta finales de la década de los ochenta, sin embargo, a partir de los años noventa su trayectoria se comportó de manera relativamente estable.

\section{Gráfica 1. Consumo nacional de las gasolinas, PIB y precios relativos} de las gasolinas en México (1960-2008)
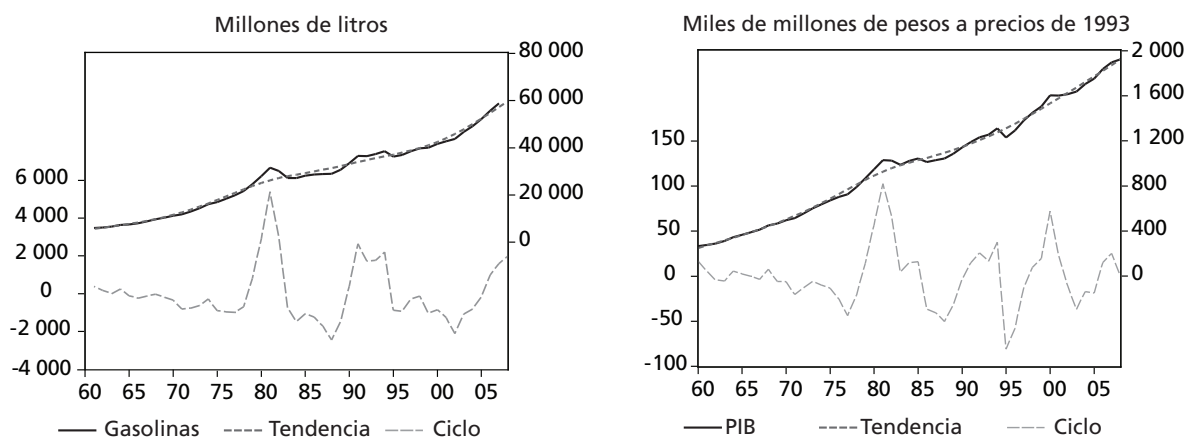

\footnotetext{
${ }^{8}$ Calculado a partir del filtro Hodrick-Prescott (HP) (Hodrick y Prescott, 1997). El filtro HP asume que una serie de tiempo se puede descomponer en una tendencia y en un ciclo.

${ }^{9}$ La tasas de crecimiento anual promedio del PIB, del consumo de gasolinas y de los precios de las gasolinas para el periodo de 1960 a 2008 fue de $4.21,5.07$ y $-1.82 \%$, respectivamente.
} 


\section{Gráfica 1. Consumo nacional de las gasolinas, PIB y precios relativos de las gasolinas en México (1960-2008) (Conclusión)}

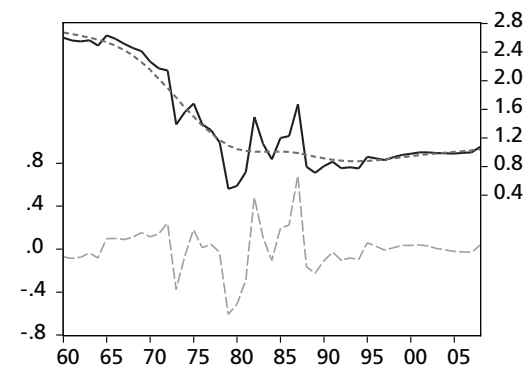

Fuente: Elaboración propia con base en la información estadística del INEGI, Pemex y Banxico.

\section{Resultados del modelo}

En principio, las estimaciones de la función de la demanda de las gasolinas se basaron en el uso de métodos econométricos que consideran el orden de integración de las series y la posible presencia del problema de la regresión espuria (Granger y Newbold, 1974, y Enders, 2004). En este sentido, el primer paso fue realizar las pruebas de raíz unitaria para identificar el orden de integración de las series (Hamilton, 1994, y Patterson, 2000). Las pruebas de hipótesis de raíces unitarias se muestran en el cuadro 2. Incluyen las pruebas de raíces unitarias: Dickey-Fuller Aumentada (ADF) (1981), Phillips-Perron (PP) (1988) y Kwiatkowsky, et al. (KPSS) (1992). El conjunto de resultados indica que la demanda de gasolina, el producto y los precios relativos de las gasolinas son series no estacionarias $\mathrm{I}(1){ }^{10}$

Considerado el orden de integración de las series, el siguiente paso fue la estimación de la posible presencia de cointegración entre las series. De esta manera se procedió a especificar modelos VAR. El número de rezagos del VAR fue seleccionado con base en el procedimiento de que cumplieran con los criterios de correcta especificación estadística, ${ }^{11}$ incluyendo la demanda de gasolinas $\left(g_{t}\right)$, el ingreso $\left(y_{\mathrm{t}}\right)$ y los precios $\left(p_{\mathrm{t}}\right)$. Así, la prueba de la raíz característica máxima de

\footnotetext{
${ }^{10}$ Cabe mencionar, que el número de retardos $(k)$ en la prueba ADF fue seleccionado de acuerdo con el procedimiento conocido como " $t$-sig" ( $\mathrm{Ng}$ y Perron, 1995), referido a la significación estadística de los retardos.

${ }^{11}$ La pruebas de diagnóstico en el modelo econométrico confirman la normalidad de los errores. Asimismo, indican que los errores no presentan problemas de autocorrelación y que no existe evidencia de heterocedasticidad.
} 
92 ECONOMÍA: TEORÍA Y PRÁCTICA • Nueva Época, número 32, enero-junio 2010

Johansen (1988 y 1992) señala que existe una relación estable de largo plazo entre el consumo de gasolinas en el ámbito nacional, el ingreso y los precios relativos de la gasolina (cuadro 3).

Cuadro 2. Pruebas de raíz unitaria

\begin{tabular}{|c|c|c|c|c|c|c|c|c|c|}
\hline \multirow{2}{*}{ Variable } & \multicolumn{3}{|c|}{$A D F$} & \multicolumn{3}{|c|}{$\mathrm{PP}(4)$} & \multicolumn{2}{|c|}{$\operatorname{KPSS}(7)$} & \multirow{2}{*}{$\begin{array}{c}\text { Orden de } \\
\text { integración }\end{array}$} \\
\hline & A & B & C & A & B & C & $\eta_{u}$ & $\eta_{t}$ & \\
\hline \multicolumn{10}{|l|}{ Niveles } \\
\hline$g_{t}$ & $-1.887(1)$ & $-1.822(1)$ & $2.581(1)$ & -1.412 & -1.961 & 4.636 & $0.173^{*}$ & $0.695^{*}$ & $\mathrm{I}(1)$ \\
\hline$y_{t}$ & $-1.534(0)$ & $-3.470(0) *$ & $3.073(1)$ & -1.551 & $-3.205^{*}$ & 4.285 & 0.180 & $0.708^{*}$ & $\mathrm{I}(1)$ \\
\hline$p_{t}$ & $-2.435(0)$ & $-2.083(0)$ & $-2.136(0)$ & -2.292 & -1.903 & $-2.019 *$ & $0.159 *$ & $0.529 *$ & $\mathrm{I}(1)$ \\
\hline \multicolumn{10}{|c|}{ Primeras diferencias } \\
\hline$\Delta g_{t}$ & $-4.049(0) *$ & $-3.799(0) *$ & $-2.421(0) *$ & $-3.917 *$ & $-3.717 *$ & $-2.917 *$ & 0.095 & 0.284 & $\mathrm{I}(0)$ \\
\hline$\Delta y_{t}$ & $-5.316(0) *$ & $-4.537(0) *$ & $-2.637(0) *$ & -5.266 * & $-4.604 *$ & $-2.433^{*}$ & 0.099 & 0.451 & $\mathrm{I}(0)$ \\
\hline$\Delta p_{t}$ & $-6.966(0) *$ & $-6.981(0)^{*}$ & $-7.012(4) *$ & $-7.442 *$ & $-7.367 *$ & $-7.352^{*}$ & 0.073 & 0.168 & $\mathrm{I}(0)$ \\
\hline
\end{tabular}

* Indica los valores que rechazan la hipótesis nula al 5\% de significación. Los valores críticos al $5 \%$ para la prueba ADF y PP, en una muestra de $t=500$, son de -3.42 incluyendo constante y tendencia (modelo A), -2.87 únicamente la constante (modelo B) y -1.95 sin constante y sin tendencia (modelo C) (Maddala y Kim, 1998). Los valores entre paréntesis representan el número de rezagos utilizados en la prueba, $\eta_{\mu}$ y $\eta_{t}$ representan los estadísticos de prueba KPSS, donde la hipótesis nula considera que la serie es estacionaria en nivel o alrededor de una tendencia determinista, respectivamente. Los valores críticos al $5 \%$ en ambas pruebas son de 0.463 y 0.146 respectivamente (Kwiatkowski, et al., 1992, p. 166). Las letras minúsculas representan el logaritmo de las series. Periodo 1960-2008.

Cuadro 3. Pruebas de cointegración basada en el procedimiento de Johansen ${ }^{12}$

\begin{tabular}{cccccc}
\hline \multicolumn{5}{c}{ Ecuación de cointegración $g_{t}=\alpha+\beta y_{t}+\gamma p_{t}+\varepsilon_{t}$} \\
\hline Hipótesis nula & $\begin{array}{c}\text { Hipótesis } \\
\text { alternativa }\end{array}$ & Traza & $\begin{array}{c}\text { Valor crítico } \\
95 \% \text { (traza) }\end{array}$ & L-max & $\begin{array}{c}\text { Valor crítico } \\
95 \% \text { (L-máx) }\end{array}$ \\
\hline$r=0$ & $r>0$ & $41.26^{*}$ & 35.19 & $22.36^{*}$ & 22.30 \\
\hline$r \leq 1$ & $r>1$ & 18.91 & 20.26 & 12.07 & 15.89 \\
$r \leq 2$ & $r>2$ & 6.84 & 9.16 & 6.84 & 9.16 \\
\hline
\end{tabular}

* Rechazo al 5\% de nivel de significación. Traza = Prueba de la traza (Mackinnon, Haug y Michelis, 1999, tabla II, p. 571). L-max = Prueba de la raíz característica máxima (Johansen, 1995, tabla 15.1, p. 214). $r$ = número de vectores de cointegración. Número de rezagos en el $\operatorname{VAR}=2$. El modelo VAR incluye las variables sin restringir: $d 74$ (dummy de pulso en 1974). Periodo $1963-2008$.

\footnotetext{
${ }^{12}$ La estimación del vector de cointegración se realizó en el paquete econométrico OxMetrics 5.00, módulo PcGive.
} 
Normalizando el vector de cointegración, asociada a la raíz característica máxima como una ecuación de la demanda de gasolinas, se obtienen los coeficientes de largo plazo (cuadro 4). Los coeficientes estimados son estadísticamente significativos y todos sus signos esperados son acordes con la teoría económica. Se observa que el consumo nacional de gasolina tiene una elasticidad ingreso positiva y mayor a la unidad. La magnitud de largo plazo de la elasticidad ingreso se encuentra dentro del rango de los estudios anteriores en otros países (Bentzen, 1994; Hunt, et al., 1999; Dahl y Kurtubi, 2001; Polemis, 2006, y Ahmadian, Chitnis y Hunt, 2007). En tanto que la elasticidad precio es negativa, con un valor de -0.28 y su magnitud es similar a las obtenidas en otras investigaciones (Hunt, et al., 1999, y Ramanathan, 1999).

Cuadro 4. Coeficientes normalizados del vector de cointegración ${ }^{a}$

\begin{tabular}{cccc}
\hline$g_{t}$ & $\alpha$ & $y_{t}$ & $p_{t}$ \\
\hline 1.000 & 10.494 & 1.004 & -0.285 \\
& $(0.860)^{*}$ & $(0.0415)^{\star}$ & $(0.0431)^{*}$ \\
\hline
\end{tabular}

Log likelihood $=210.8$

a Todas las variables están expresadas en logaritmos. Los números entre paréntesis son los errores estándar. * Indica rechazo al 5\% de nivel de significación. Periodo 1963-2008.

De acuerdo con los resultados, la elevada elasticidad ingreso se debe en alguna medida al incremento de las necesidades de transporte de la población y de los sectores económicos. Así como el hecho de que el auto privado se ha convertido en un elemento fundamental en las sociedades urbanas. En este contexto, el resultado de la elasticidad ingreso sugiere que el crecimiento del transporte en México, asociado con el crecimiento económico, es aún intensivo en el uso de gasolinas. De igual manera, se debe considerar que la elasticidad ingreso incide en el consumo a partir de la composición y del crecimiento de la flota vehicular. En efecto, el fuerte efecto de los ingresos en el consumo de gasolinas se puede producir a partir de su influencia en el nivel de las características de los automóviles y de su repercusión en el crecimiento de la cantidad de vehículos (Wheaton, 1982). Así, para reducir el consumo de gasolinas es importante contener el crecimiento rápido de la flota vehicular.

En contraste con el efecto de los ingresos, la influencia del precio en el modelo se encuentra limitada. Efectivamente, las estimaciones indican que la 
demanda de gasolinas es poco sensible al movimiento en precios. Por tanto, los aumentos en los precios no desalientan el consumo de gasolina ni tampoco ayuda a su utilización de manera más eficiente (Galindo y Salinas, 1997). No obstante, el valor específico hallado es de particular relevancia para concretar una estrategia bien definida sobre el uso de los instrumentos económicos. En efecto, una baja elasticidad precio indica que es necesario una modificación importante en los precios para obtener una reducción sustancial en el consumo de las gasolinas. De hecho, es difícil instrumentar una política energética basada únicamente en los precios de los combustibles por sus consecuencias económicas y sus repercusiones en los consumidores.

Por otra parte, la evidencia empírica sugiere que es posible identificar una trayectoria de equilibrio de corto plazo entre el nivel de la actividad económica y la relación de precios de las gasolinas respecto al consumo de gasolinas en México. En este sentido, se estimó un ECM (Engle y Granger, 1987) a fin de obtener respuestas en el corto plazo. El cuadro 5 muestra los resultados de la estimación del ECM de la demanda de gasolinas. Cada coeficiente de las variables denota la elasticidad a corto plazo.

Los resultados del ECM son consistentes atendiendo a las pruebas de correcta especificación. Ello indica que el modelo contiene toda la información sistemática pertinente al fenómeno, dado el conjunto de información disponible. De esta manera, el modelo permite que se pueda utilizar para realizar pronósticos con distintos escenarios en las variables exógenas. De hecho, los coeficientes resultan acordes con la teoría económica y son estadísticamente significativos. La elasticidad ingreso de corto plazo está por debajo de la unidad y se estima en 0.721 , lo que implica que un aumento de $1 \%$ del PIB aumentaría la demanda de gasolina a un ritmo más lento $(0.72 \%)$. La elasticidad de corto plazo respecto al precio es cercana a cero (-0.041); este resultado confirma la evidencia de que la elasticidad del precio de la demanda de las gasolinas es baja en el corto plazo (Dahl y Sterner, 1991b). Así, por ejemplo, la respuesta de la demanda de gasolina a fluctuaciones en sus precios revela la dificultad de los consumidores para sustituir las gasolinas con otros productos energéticos. Por otra parte, se observa que los valores proyectados por el modelo representan satisfactoriamente los valores observados de las variaciones en la demanda de gasolina, con un grado de ajuste de $75 \%$, en tanto que el mecanismo de corrección de error es negativo y significativo. Por tanto, los desajustes en la relación de equilibrio son incorporados en la modelación de corto plazo. La velocidad de ajuste del modelo es de $13.3 \%$ para cada año. 
Cuadro 5. Resultados del ECM ${ }^{13}$

\begin{tabular}{|c|c|}
\hline Variables & $\Delta g_{t}$ \\
\hline$\Delta y_{\mathrm{t}}$ & $0.7212(0.1235)^{a}$ \\
\hline$\Delta p_{\mathrm{t}}$ & $-0.0414(0.0184)$ \\
\hline$\Delta g_{\mathrm{t}-1}$ & $0.2383(0.0808)$ \\
\hline$u_{t-1}$ & $-0.1333(0.0675)$ \\
\hline$d_{t 82}$ & $-0.0672(0.0251)$ \\
\hline \multicolumn{2}{|l|}{ Estimación por OLS } \\
\hline$R^{2}$ & 0.759 \\
\hline$R^{2}$ ajustada & 0.736 \\
\hline$D W$ & 1.868 \\
\hline Jarque-Bera & $\chi^{2}=2.544[0.284]$ \\
\hline$L M$ (2 rezagos) & $F=1.737[0.189]$ \\
\hline$A R C H$ (2 rezagos) & $F=0.695[0.504]$ \\
\hline
\end{tabular}

a Los números entre paréntesis de los coeficientes representan el error estándar. $R^{2}$ es el coeficiente de determinación. DW es el estadístico Durbin-Watson para autocorrelación. La normalidad se prueba mediante el estadístico Jarque-Bera. LM (Multiplicadores de Lagrange) y ARCH (heterocedasticidad condicional autorregresiva) son pruebas para autocorrelación y heterocedasticidad, respectivamente, con un marginal de significación entre corchetes. $\mathrm{d}_{\mathrm{t} 82}$ (dummy de pulso en 1982). Periodo 1962-2008.

Finalmente, en el cuadro 6 se presentan las elasticidades ingreso y precio de corto y largo plazos. Los resultados de la magnitud de las elasticidades son consistentes con las estimaciones reportadas en diversas investigaciones empíricas internacionales respecto a la sensibilidad del consumo de gasolinas a cambios en el nivel de la actividad económica y en los precios (D. J. Graham y S.Glaister, 2002 y 2004, y P. B. Goodwin, et al., 2004). También, en buena medida, son estimaciones razonables y similares a estudios que se han realizado para México (E. R.Berndt y J. Botero, 1985; L. M. Galindo y E. Salinas, 1997; N. Wohlgemuth, 1997; y L. M. Galindo, 2005). ${ }^{14}$

\footnotetext{
${ }^{13}$ La estimación del MCE se realizó en el paquete econométrico EViews 6.0.

${ }^{14}$ Un enfoque alternativo para calcular las elasticidades precio e ingreso es mediante un modelo ARDL. Las elasticidades de corto y largo plazos del precio e ingreso obtenidos con este método son: $-0.07,-0.17,0.46$ y 1.06 , respectivamente (Reyes, 2009).
} 
Cuadro 6. Elasticidades ingreso y precio de corto y largo plazos de la demanda de gasolinas

\begin{tabular}{lcc}
\hline & Corto plazo & Largo plazo \\
\hline Elasticidad ingreso & 0.721 & 1.004 \\
Elasticidad precio & -0.041 & -0.285 \\
\hline
\end{tabular}

Fuente: Elaboración propia con base en las estimaciones de los cuadros 4 y 5 .

\section{Pronósticos de la demanda de gasolinas}

La elaboración de pronósticos con base en modelos econométricos no es una actividad sencilla. La teoría econométrica sobre la elaboración de pronósticos establece que el uso de modelos sin problemas de especificación, con parámetros constantes y estimados con series estacionarias, permite obtener resultados óptimos. Sin embargo, es común que se desconozca la especificación correcta y que las series no sean estacionarias con cambio estructural. Este documento utilizó una combinación de técnicas econométricas con la obtención de modelos causales con sentido económico que permitieran obtener pronósticos relativamente razonables.

En este contexto, utilizando el filtro HP y la distribución de frecuencias de las tasas de crecimiento del PIB (gráfica 2), se puede apreciar el crecimiento probable de largo plazo de la economía. Éste se ubica alrededor de 3.6\% anual, y muy probablemente cambiará ligeramente en los próximos años.

Asimismo, con base en un modelo de series de tiempo y utilizando la información del crecimiento potencial, se realizó un pronóstico del PIB para el periodo de 2009 a 2020. Este procedimiento es útil para realizar una gráfica de Fan Chart. ${ }^{15}$ De esta manera, la línea punteada en la gráfica 3 representa el escenario base con mayor probabilidad de ocurrencia que se ubica en $3.6 \%$ anual. También, se pueden observar las bandas de probabilidad ajustadas al 75, 85 y $95 \%$ en escala de grises oscuro, menos oscuro y más claro respectivamente.

\footnotetext{
${ }^{15}$ La estimación de los modelos econométricos permite construir distintos escenarios sobre las trayectorias del PIB, e incorporar mediante el uso de Fan Chart el pronóstico base y sus bandas de probabilidad asociadas con los escenarios que presentan una mayor probabilidad de ocurrencia. El Fan Chart representa la función de probabilidades de los valores futuros de una variable, condicionada la información conocida en el presente (Britton, et al., 1998). Esta metodología se utiliza con el propósito de mostrar explícitamente que las proyecciones se enmarcan inevitablemente en una situación de incertidumbre y riesgos diversos, no necesariamente simétricos.
} 
Gráfica 2. Trayectoria de la economía mexicana (1960-2008)

Tasa de crecimiento del PIB

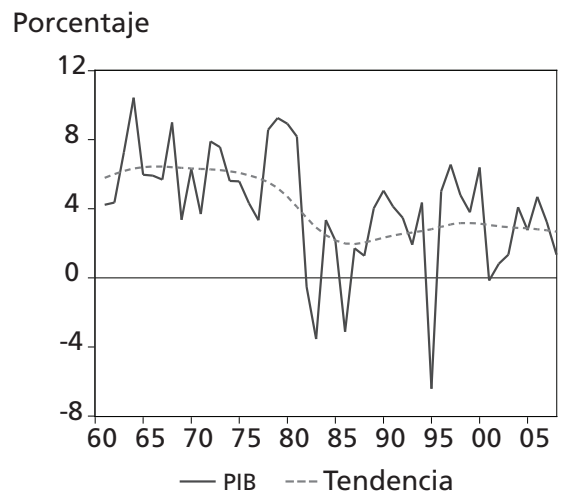

Crecimiento potencial de la economía (distribución de frecuencias)

Frecuencia relativa

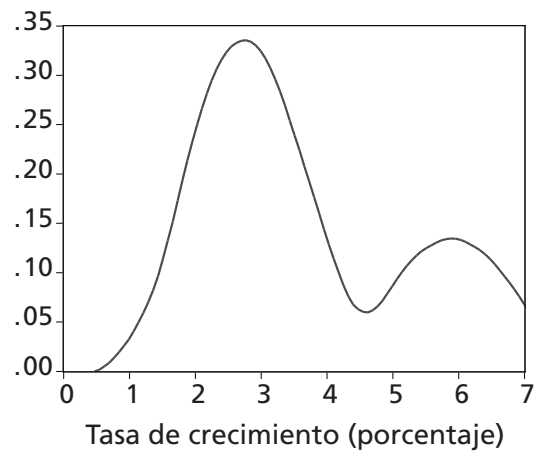

Fuente: Elaboración propia con base en información estadística del INEGI.

Gráfica 3. Proyecciones del PIB (2009-2020) y bandas de probabilidad (Fan Chart)

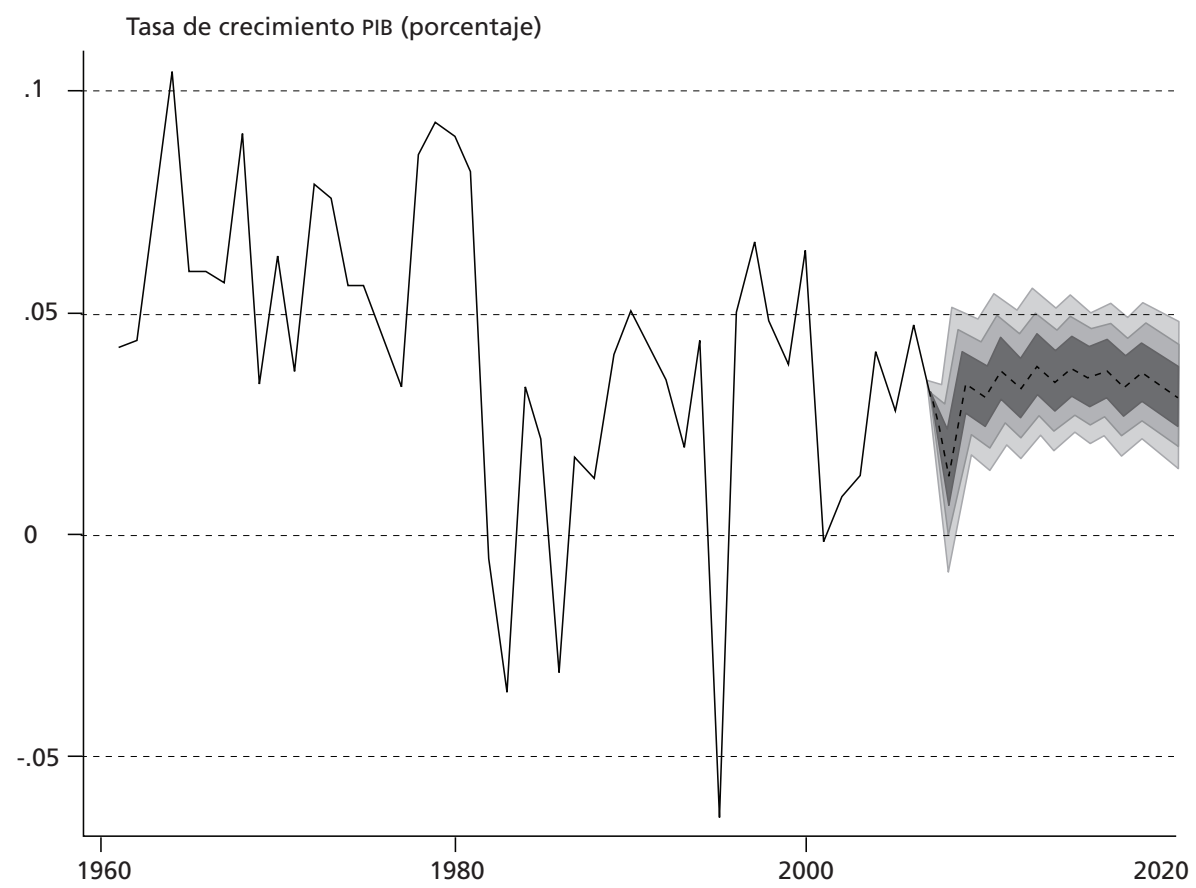

Fuente: Elaboración propia. 
Así, el escenario con mayor probabilidad sitúa al crecimiento de la economía mexicana entre 2.6 y $4.6 \%$ anual. De igual manera, bajo el supuesto de que no se presenten cambios estructurales, la tasa de crecimiento debería ubicarse cerca de $3.6 \%$ anual. Cabe señalar que existe $25 \%$ de probabilidad de que el crecimiento económico en México se ubique por arriba de estos rangos.

En lo que se refiere a los precios de las gasolinas, en el escenario base o inercial (Bussines As Usual - BAU), se supone que los precios permanecen constantes. Ello indica que los precios de las gasolinas se incrementan en una proporción similar al incremento en el nivel general de precios. No obstante, como ejercicio ilustrativo, se utilizaron precios altos (tasa de crecimiento anual de $5.5 \%) .{ }^{16}$ Lo señalado, junto con las proyecciones del PIB, se toma como los escenarios para la construcción de las proyecciones de la demanda de gasolinas. En este sentido se construyeron cuatro escenarios: bajo, moderado, base y alto, y se presentan en el cuadro 7:

Cuadro 7. Parámetros estimados (2009-2020).

Tasa de crecimiento anual promedio

(Porcentaje)

\begin{tabular}{lccccc}
\hline Tipo de escenario & \multicolumn{2}{c}{ Precios base } & \multicolumn{2}{c}{ Precios altos } \\
& PIB & Gasolina & Diesel & Gasolina & Diesel \\
\hline Bajo & 2.6 & & & 5.5 & 5.5 \\
Moderado & 3.6 & & & 4.0 & 4.0 \\
Base & 3.6 & 2.3 & 2.3 & & \\
Alto & 4.6 & 2.3 & 2.3 & \\
\hline
\end{tabular}

Fuente: Elaboración propia.

Las proyecciones de la demanda de gasolinas con base en los resultados del ECM, en el ámbito nacional del año 2009 hasta el 2020 comparando cuatro trayectorias distintas se pueden apreciar en la gráfica 4. La tasa promedio anual de crecimiento del consumo de gasolinas con un escenario base es de $3.7 \%$, la cual se incrementa a $4.9 \%$ con un escenario alto. Con un escenario bajo y moderado, el consumo de gasolinas en México presenta un crecimiento anual promedio de 1.5 y $2.8 \%$, respectivamente.

\footnotetext{
${ }^{16}$ Con base en la iniciativa del gobierno mexicano de incrementar $5.5 \%$ el precio de gasolinas a partir de 2008 (Paz, 2008).
} 


\section{Gráfica 4. Trayectoria e incertidumbre en la predicción de demandas de gasolinas (2009-2020)}
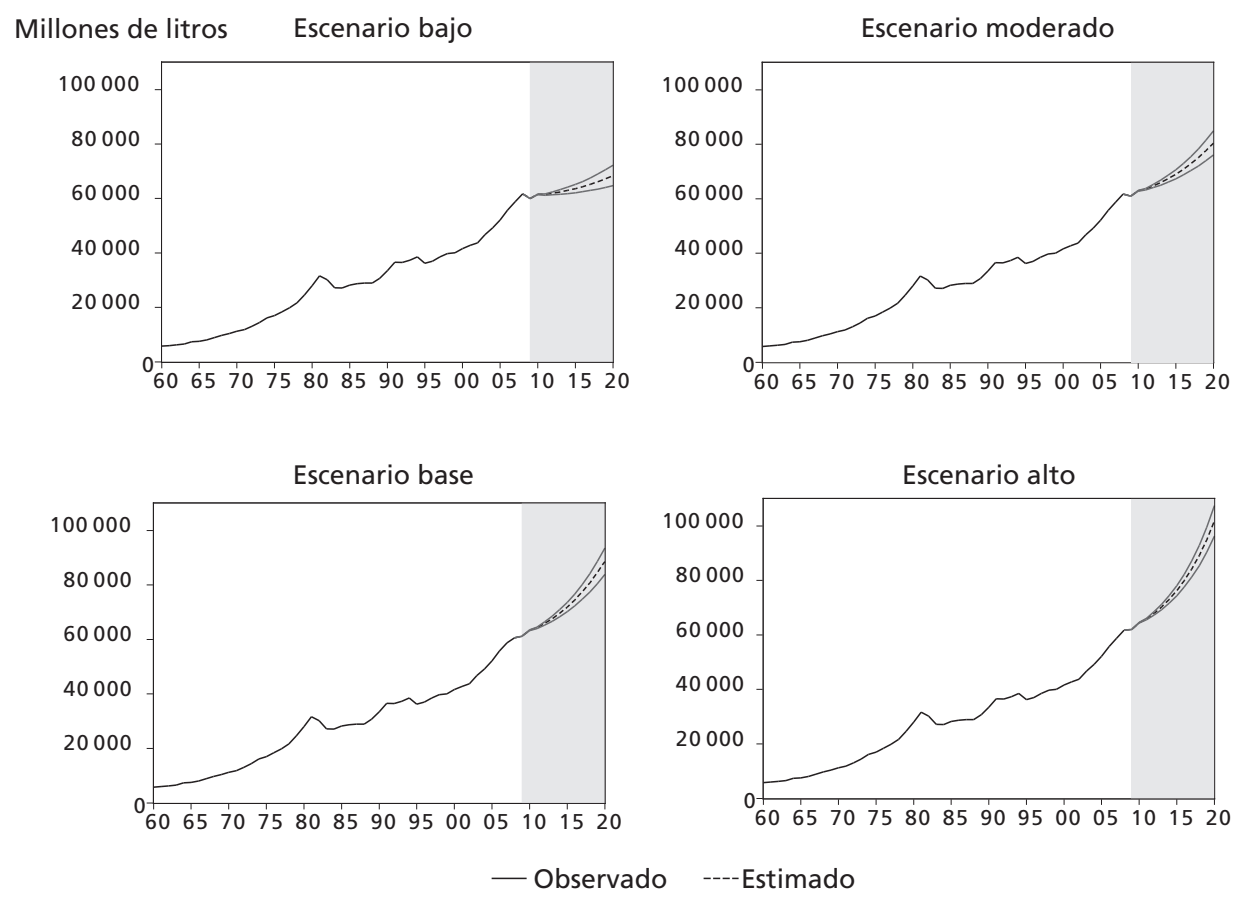

Fuente: Elaboración propia.

Los resultados de las proyecciones muestran que bajo las condiciones actuales, y de no registrarse cambios significativos en la política de precios, es decir, bajo un escenario base, la demanda de gasolinas tiende a incrementarse. También los resultados indican que un aumento en el precio de las gasolinas no es suficiente para controlar el consumo de gasolinas y que este consumo continuará elevándose asociado con el crecimiento económico. De esta forma, la evolución del consumo de combustibles y su relación con las emisiones de $\mathrm{CO}_{2}$ será fundamental para los impactos ambientales, destacando las implicaciones de políticas públicas (Zachariadis y Kouvaritakis, 2003).

\section{Las emisiones de $\mathrm{CO}_{2}$ y el uso de gasolinas en el autotransporte}

El consumo energético del sector transporte procede fundamentalmente de los combustibles fósiles y está asociado con los principales impactos negativos del 
transporte: cambio climático, contaminación atmosférica y problemas de salud (Calthrop y Proost, 1998; Kayser, 2000; Lenz, Prüller y Gruden, 2003; Sperling, 2004; y Stanley, Hensher y Loader, 2009). De hecho, en los años recientes la evidencia empírica ha abordado cada vez más las diversas consecuencias ambientales del consumo de gasolinas, en particular respecto a las emisiones de GEI (Weiss, et al., 2003; Lutsey y Sperling, 2007; y Bandivadekar, 2008). Esto ha conducido a desarrollar distintas estrategias y medidas a fin de reducir el nivel de emisiones de GEI (Litman, 2005; Kosugi, Tokimatsub y Yoshida, 2005; y Schafer y Jacoby, 2006).

En 2008 la gasolina y el diesel fueron los combustibles más utilizados en el sector transporte en México; su consumo se sitúa alrededor de $89 \%$ del total de combustibles consumidos, y equivale a $97.7 \%$ del utilizado en el transporte por carretera. En el periodo de 1980 a 2008 se observó un incremento en el consumo de las gasolinas en el sector automotor. Destaca el consumo de gasolina, que registró una tasa anual promedio de 3.39\% y provocó que su participación relativa aumentara de $65 \%$ en 1980 a $74 \%$ en 2008. Por lo contrario, el consumo de diesel creció a una tasa de $1.67 \%$, reduciendo su participación relativa $10 \%$, al pasar de una participación de 35\% en 1980 a 25\% en 2008 (gráfica 5). En 1995 se presentó un descenso en el consumo de estas gasolinas debido a la crisis económica que enfrentó México, en la que el PIB sufrió una caída de 6.21\% respecto al año anterior. ${ }^{17}$ No obstante, a partir de 1996 el consumo de gasolinas ha aumentado de forma paulatina.

Gráfica 5. Consumo nacional de gasolinas en el sector automotor (1960-2008)
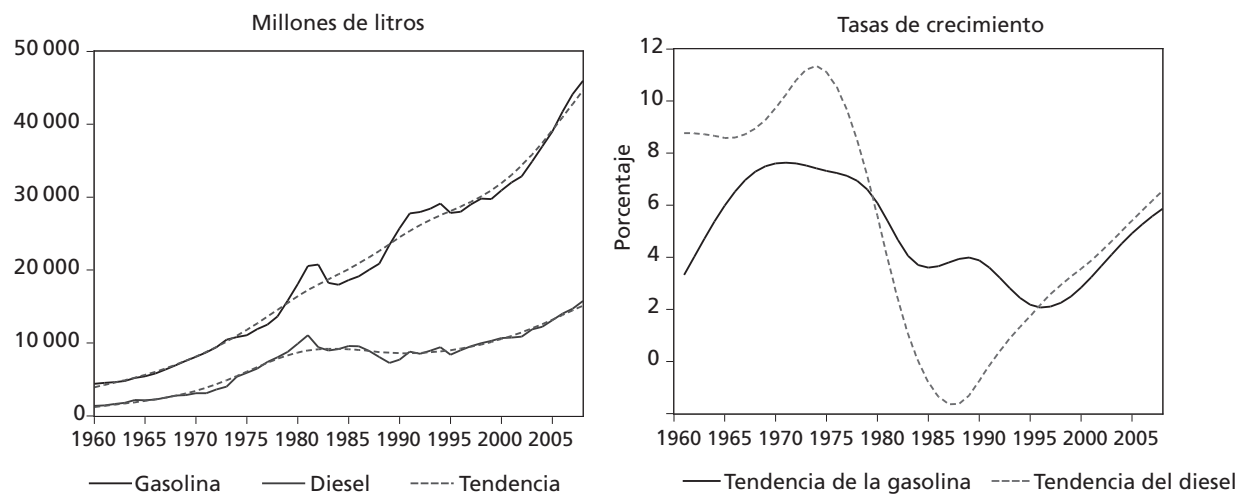

Fuente: Elaboración propia con base en la información estadística de Pemex.

${ }^{17}$ Esta contracción en la demanda de gasolinas también se origina por una alza severa de los precios (Uri y Boyd, 1999). 
Por otra parte, las emisiones en UE de $\mathrm{CO}_{2}$ producidas en el 2007 por el sector transporte a partir de la quema de combustibles fósiles sumaron 149221 $\mathrm{Gg}$, de las cuales las emisiones del sector autotransporte representaron $90.87 \%$ $(135591 \mathrm{Gg}) .{ }^{18} \mathrm{El}$ crecimiento sostenido de la flota vehicular ha sido el principal factor para el aumento en el consumo de gasolina y diesel y, por consiguiente, del incremento en el impacto sobre el ambiente, al emitir mayor cantidad de contaminantes y GEI (Secretaría de Energía, 2008). En 2007, de la flota vehicular total existente, $94.6 \%$ funcionaba con gasolina, $4.3 \%$ con diesel, y el porcentaje restante con gas LP, y en menor medida con gas natural comprimido.

Otro asunto importante en este contexto es la relación entre las emisiones de GEI y el consumo de combustible, ya que el $\mathrm{CO}_{2}$ es la principal fuente de emisiones de GEI de un automóvil y el nivel de emisiones de $\mathrm{CO}_{2}$ de los automóviles está directamente relacionado con el consumo de combustibles.

Las emisiones de $\mathrm{CO}_{2}$ provenientes del diesel resultan en un mayor factor de emisión ${ }^{19}$ que el de la gasolina. Considerando que en el cálculo de las emisiones de $\mathrm{CO}_{2}$, los factores de emisión están basados en el contenido de carbono del combustible, ${ }^{20}$ para 2008 los motores de gasolina y diesel emitieron en promedio 2.248 y $2.867 \mathrm{~kg}$ de $\mathrm{CO}_{2}$, por cada litro quemado, respectivamente. ${ }^{21}$ Por tanto, la quema de gasolina en los vehículos emite más $\mathrm{CO}_{2}$ que el diesel, no por su composición química, sino por la cantidad de litros que se consumen (Sullivan, et al., 2004).

De esta forma, el promedio anual de las emisiones estimadas de $\mathrm{CO}_{2}$ del consumo de gasolinas de 2008 hasta 2020, con base en los factores de emisión y en los pronósticos de los distintos escenarios del consumo de gasolinas, se presenta en la gráfica 6. Los GEI del autotransporte están expresados en unidades de $\mathrm{Gg}$ de $\mathrm{UE}$ de $\mathrm{CO}_{2}$ y se asume un promedio de 2.6 toneladas de $\mathrm{CO}_{2}$ por 1000 litros de gasolinas.

\footnotetext{
${ }^{18}$ Cálculos propios a partir de las estadísticas de la Sener (Series del Balance Nacional de Energía 1965-2007).

${ }^{19}$ Corresponde a la unidad de conversión para estimar emisiones a partir de datos de actividad.

${ }^{20} \mathrm{El}$ factor de emisión de la gasolina es $69300 \mathrm{~kg} / \mathrm{TJ}\left(\mathrm{TJ}=\right.$ terajules, $1 \mathrm{TJ}=10^{12}$ joules $)$ y para el diesel es $74100 \mathrm{~kg} / \mathrm{TJ}$ (IPCC, 2006). Las gasolinas están compuestas por moléculas que agrupan átomos de carbono e hidrógeno ordenados en formas de cadenas; la gasolina utiliza el octano $\left(\mathrm{C}_{8} \mathrm{H}_{18}\right)$, mientras que el diesel utiliza el cetano $\left(\mathrm{C}_{16} \mathrm{H}_{34}\right)$. Cuando el combustible se quema, el carbono se une al oxígeno del aire para formar $\mathrm{CO}_{2}$.

${ }^{21}$ Suponiendo que el sector automotor en México aproximadamente utiliza 7 litros de gasolina y 6 litros de diesel por cada $100 \mathrm{~km}$. En promedio, se emiten 163.38 gramos de $\mathrm{CO}_{2}$ por kilómetro recorrido.
} 


\section{Gráfica 6. Promedio anual de $\mathrm{CO}_{2}$ por consumo de gasolinas} con distintos escenarios (2008-2020)

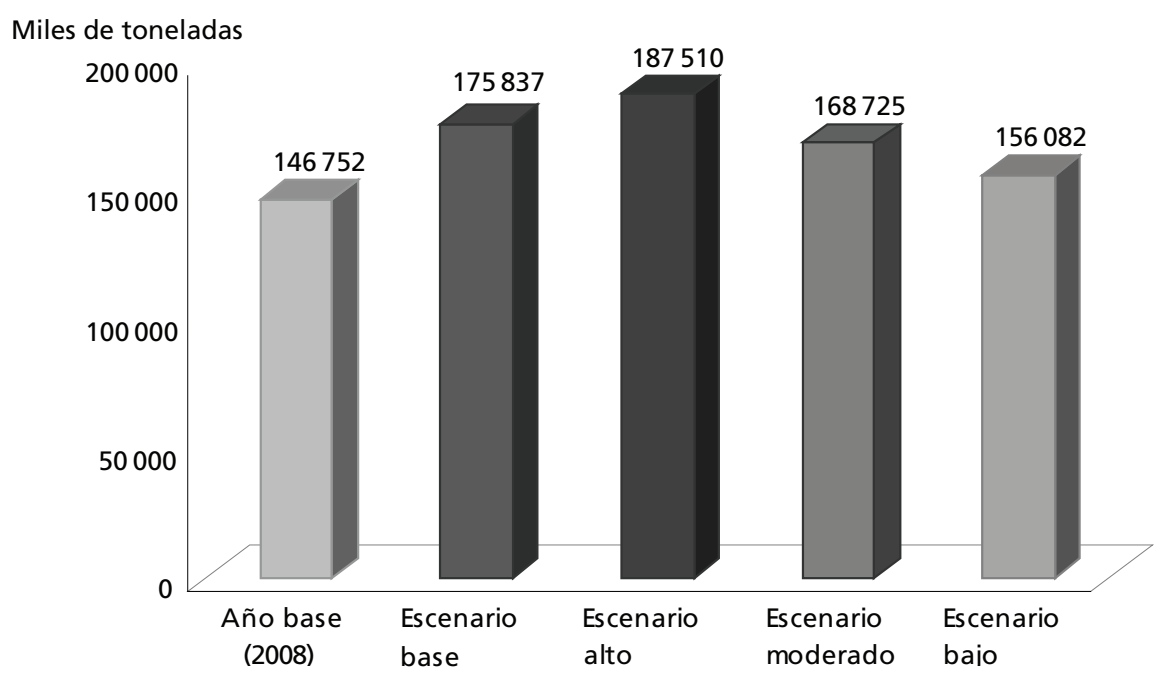

Fuente: Elaboración propia.

Los resultados señalan que en el escenario más probable de un crecimiento económico continuo con un consumo de gasolinas similar al comportamiento histórico, las emisiones de $\mathrm{CO}_{2}$ a la atmósfera tenderán a crecer considerablemente $^{22}$ en los próximos años, En efecto, el comportamiento histórico sugiere que la demanda de gasolinas no va a disminuir. Esto indica que es necesario instrumentar una estrategia que incluya medidas sustanciales para separar el crecimiento económico de las emisiones de $\mathrm{CO}_{2}$ a la atmósfera. Las simulaciones confirman que sólo con el escenario de bajo crecimiento económico y precios altos es posible reducir las emisiones de $\mathrm{CO}_{2}$ a la atmósfera. Sin embargo, este escenario no es económica ni socialmente viable. Es importante destacar que el sector autotransporte representa el sector más importante de cara al desarrollo de políticas encaminadas a la reducción de emisiones de GEI. ${ }^{23}$

\footnotetext{
${ }^{22}$ Las emisiones de GEI correspondiente al $\mathrm{CO}_{2}$ provenientes de la quema de gasolinas en México ascendieron en 2002 a $101843 \mathrm{Gg}$, mientras que para el año base (2008) se situaron en $146752 \mathrm{Gg}$, con un crecimiento anual promedio de $6.28 \%$. Para 2020, considerando el escenario base, se emitirán $175837 \mathrm{Gg}$ anuales promedio, con una tasa de crecimiento anual promedio de 3.45 por ciento.

${ }^{23}$ Además de contribuir con emisiones de gases con efecto invernadero (gases globales), los vehículos emiten gases que son contaminantes locales (por ejemplo el ozono) y regionales (principalmente las emisiones de NOx), Burón, et al. (2004).
} 
La opción más razonable es un cambio significativo en el modelo de transporte, responsable de más de $65 \%$ del consumo de los productos derivados del petróleo (Secretaría de Energía, 2008).

Es indudable que la reducción de las emisiones de $\mathrm{CO}_{2}$ provenientes del sector transporte por carretera es de fundamental importancia para enfrentar los impactos del cambio climático sobre la sociedad mexicana. Considerando que la mayoría de las estimaciones de $\mathrm{CO}_{2}$ por el consumo de gasolinas tienden a crecer considerablemente, es evidente que las acciones que concrete el gobierno de México para alcanzar los compromisos específicos para la reducción de emisiones de GEI, resultarán una tarea enorme. Definitivamente, sólo se lograrán la metas de reducción de emisiones con mayor disponibilidad social y recursos económicos, y esto dependerá de la inversión en tecnología avanzada para mitigar la emisiones de GEI.

\section{CONCLUSIONES Y OTRAS CONSIDERACIONES}

La reducción de emisiones contaminantes y el mantenimiento del bienestar de las sociedades requieren un delicado equilibrio entre políticas que muchas veces tienen efectos contrarios (Martín, et al., 2007). En el caso del $\mathrm{CO}_{2}$, esta cuestión es esencial porque estas emisiones dependen del consumo de combustibles fósiles. Las políticas encaminadas a la reducción de emisiones pueden contraponerse con los objetivos de desarrollo y crecimiento de la sociedad. Esto indica que es necesario que se instrumenten diversas medidas para buscar separar el crecimiento económico del consumo de combustibles y de las emisiones de $\mathrm{CO}_{2}$ asociadas.

En este sentido, la definición adecuada de una estrategia para México requiere del conocimiento puntual de las diversas trayectorias de las emisiones de $\mathrm{CO}_{2}$ en el futuro inmediato. El autotransporte en México es responsable de cerca de $20 \%$ de las emisiones de $\mathrm{CO}_{2}$ relacionadas con el consumo humano de energía. Si los factores que generan la actividad del transporte continúan, las emisiones de $\mathrm{CO}_{2}$ podrían incrementarse más de $50 \%$ sobre el nivel actual en 2020 (año base de referencia, 2008). En efecto, dada la dependencia actual de los combustibles por el sector transporte, necesariamente esto da lugar a un aumento considerable de las emisiones de $\mathrm{CO}_{2}$. En este sentido, es necesario estimular y desarrollar medidas que ayuden a revertir las tendencias actuales de consumo energético del sector transporte. 
Los resultados obtenidos muestran que el consumo de gasolinas es particularmente sensible a la trayectoria del ingreso y poco sensible a los precios relativos. Esto tiene, en principio, consecuencias negativas sobre el cambio climático e implica limitantes importantes en el uso de los precios como instrumento económico para regular el consumo de gasolinas en México. De hecho, una política de precios por sí sola no resuelve el problema. No obstante, podría ser exitosa en el control de la contaminación de la atmósfera si está necesariamente acompañada de otro tipo de políticas públicas.

Por tanto, habría que evaluar distintas medidas que puedan ayudar a limitar el crecimiento en el uso de las gasolinas e impulsar la reducción de las emisiones de $\mathrm{CO}_{2}$ del sector transporte por carretera en México. Entre estas opciones se encuentran: la modernización de la flota vehicular ${ }^{24}$ que mejore su eficiencia energética y reduzca su emisión de gases contaminantes (Schafer y Jacoby, 2006); un consumo óptimo de combustibles fósiles, por medio de un reordenamiento urbano que disminuya el número de viajes y los kilómetros recorridos, y de un mejoramiento considerable del transporte público que eleve su capacidad de sustituir al transporte privado, ${ }^{25}$ una mejora en las gasolinas; ${ }^{26}$ el establecimiento de programas eficaces de verificación de emisiones contaminantes provenientes de vehículos con gasolina y diesel, en especial en zonas urbanas e industriales donde la exposición de la población es mayor y muy importante; ${ }^{27}$ y una campaña de información mediática importante dirigida a convencer a los ciudadanos de las bondades del servicio de transporte público, que ponga el acento en los daños que se producen en el ambiente a partir de su elección en el modo de transporte (Shafer y Victor, 1999).

\footnotetext{
${ }^{24}$ Ésta requiere la renovación acelerada de la flota vehicular que incluya el retiro o el reemplazo. Por ejemplo, la ampliación de la flota vehicular de motores a diesel provoca un mayor ahorro de combustible (Schipper, et al., 2002) y reduce considerablemente las emisiones de $\mathrm{CO}_{2}$ (Zervas, et al., 2006).

${ }^{25} \mathrm{El}$ mejoramiento en las redes de transporte debe incluir acciones, como la expansión del metro, la creación de trenes suburbanos, el establecimiento de corredores viales y el transporte colectivo (por ejemplo, el transporte exclusivo para escuelas y universidades).

${ }^{26}$ Es indudable que los combustibles más limpios deben ser parte integral de un fuerte control de la contaminación de los vehículos automotores con gasolina y diesel.

${ }^{27}$ En México, la mayoría de las estrategias de control de emisiones que se han implantado tratan de resolver el problema en lo local. Así, estas estrategias han modificado tanto las especificaciones de los combustibles como la cantidad de GEI que emiten. Sin embargo, el hecho de que algunos gases tengan diferentes efectos, ocasiona que en el diseño de estrategias de control de la contaminación tengan interacciones en tres escalas: local, regional y global (L. Díaz y J. Gasca, 2004).
} 
Además, se requiere del cumplimento de diversos compromisos de los programas de cambio climático. ${ }^{28}$ No obstante, éstas son herramientas de aplicación que quedan fuera de este análisis. Desde luego, los análisis que consideren estas medidas permitirán profundizar, validar o incluso rectificar algunos de los resultados aquí obtenidos.

\section{REFERENCIAS BIBLIOGRÁFICAS}

Ahmadian, M., M. Chitnis, y L. C. Hunt (2007), "Gasoline Demand, Pricing Policy and Social Welfare in the Islamic Republic of Iran”, OPEC Review, 31 (2), páginas 105-124.

Akinboade, O., E. Ziramba, y W. L. Kumo (2008), "The Demand for Gasoline in South Africa: An Empirical Analysis Using Co-integration Techniques", Energy Economics, 30 (6), pp. 3222-3229.

Alves, D., y R. Bueno (2003), "Short-Run, Long-Run and Cross Elasticities of Gasoline Demand in Brazil", Energy Economics, 25 (2), pp. 191-199.

Austin D. (2008), "Climate-Change Policy and $\mathrm{CO}_{2}$ Emissions from Passenger Vehicles", Congressional Budget Office, Economic and Budget Issue Brief, octubre, pp. 1-8.

Baltagi, B. H., y J. M. Griffin (1983), "Gasoline Demand in the OECD: An Application of Pooling and Testing Procedures”, European Economic Review, 22 (2), páginas $117-137$.

Banaszak, S., U. Chakravorty, y P. Leung (1999), "Demand for Ground Transportation Fuel and Pricing Policy in Asian Tigers: A Comparative Study of Korea and Taiwan", The Energy Journal, 20 (2), pp. 145-165.

Bandivadekar, A., et al. (2008), "Reducing the Fuel Use and Greenhouse Gas Emissions of the Us Vehicle Fleet", Energy Policy, 36 (7), pp. 2754-2760.

Basso, J. L., y T. H. Oum (2007), "Automobile Fuel Demand: A Critical Assessment of Empirical Methodologies", Transport Reviews, 27 (4), pp. 449-484.

Bentzen, J. (1994), "An Empirical Analysis of Gasoline Demand in Denmark Using Cointegration Techniques", Energy Economics, 16 (2), pp. 139-143.

y T. Engsted (1999), "A Revival of the Autoregressive Distributed Lag Model in Estimating Energy Demand Relationships”, Energy, 26 (1), pp. 45-55.

\footnotetext{
${ }^{28}$ La implantación de estándares rigurosos para recortar las emisiones e incrementar el rendimiento de las gasolinas daría como resultado una disminución considerable de las emisiones de los vehículos, independientemente de que aumenten o no los precios de las gasolinas (Austin, 2008).
} 
Berndt, E. R., y G. Botero (1985), "Energy Demand in the Transportation Sector of Mexico", Journal of Development Economics, 17 (3), pp. 219-238.

Blanchard, O. (1997), "Is There a Core of Usable Macroeconomics?", The American Economic Review, 87 (2), pp. 244-246.

Britton, E., P. Fisher, y Whitley (1998), “The Inflation Report Projections: Understanding the Fan Chart", Bank of England Quarterly Bulletin, 38 (1), pp. 30-37.

Burón, J. M., F. Aparicio, O. Izquierdo, A. Gómez, e I. López (2004), “Estimation of the Input Data for the Prediction of Road Transportation Emissions in Spain from 2000 to 2010 Considering Several Scenarios", Atmospheric Environment, 39 (30), pp. 5585-5596.

Calthrop, E., y S. Proost (1998), "Road Transport Externalities. Interaction Between Theory and Empirical Research", Environmental and Resource Economics, 11 (3-4), pp. 335-348.

Chandrasiri, S. (2006), "Demand for Road-Fuel in a Small Developing Economy: The Case of Sri Lanka”, Energy Policy, 34 (14), pp. 1833-1840.

Cheung, K., y E. Thomson (2004), "The Demand for Gasoline in China: A Cointegration Analysis", Journal of Applied Statistics, 31 (5), pp. 533-544.

Dahl, C. A. (1986), “Gasoline Demand Survey”, The Energy Journal, 7 (1), pp. 67-82. y T. Sterner (1991a), "A Survey of Econometric Gasoline Demand Elasticities", International Journal of Energy Systems, 11 (2), pp. 53-76.

— y T. Sterner (1991b), “Analysing Gasoline Demand Elasticities: A Survey”, Energy Economics, 13 (3), pp. 203-210.

— y Kurtubi (2001), "Estimating Oil Product Demand in Indonesia Using a Cointegrating Error Correction Model", OPEC Review, 25 (1), pp.1-25.

De Vita, G., K. Endresen, y L. C. Hunt (2006), An Empirical Analysis of Energy Demand in Namibia, Energy Policy, 34 (18), pp. 3447-3463.

Díaz, L., y J. Gasca (2000), INEGEI. Energía: Sector transporte (2000-2001), Instituto Mexicano del Petróleo (IMP), estudio elaborado para el Instituto Nacional de Ecología (INE).

Dickey, D. A., y W. A. Fuller (1981), "Likelihood Ratio Statistics for Autoregressive Time Series with a Unit Root", Econometrica, 49 (4), pp. 1057-1072.

Drollas, L. (1984), “The Demand for Gasoline: Further Evidence”, Energy Economics, 6 (1), pp. 71-82.

Eltony, M. N., y N. H. Al-Mutairi (1995), "Demand for Gasoline in Kuwait: An Empirical Analysis Using Cointegration Techniques”, Energy Economics, 17 (3), pp. 249-253. 
Enders, W. (2004), Applied Econometrics Time Series, Wiley Series in Probability and Statistics, 2a edición.

Engle, R. F., y C. W. J. Granger (1987), “Cointegration and Error Correction: Representation Estimation and Testing”, Econometrica, 55 (2), pp. 251-276.

Eskeland, G., y T. Feyzioglu (1997), "Is Demand for Polluting Goods Manageable? An Econometric Study of Car Ownership and Use in Mexico", Journal of Development Economics, 53 (2), pp. 423-445.

Espey, M. (1998), "Gasoline Demand Revisited: An International Meta-Analysis of Elasticities”, Energy Economics, 20 (3), pp. 273-295.

Galindo, L. M., y E. Salinas (1997), "La demanda de gasolinas en México: La condición de exogeneidad y el comportamiento de los agentes económicos”, en INE-Semarnat (comp.), Instrumentos económicos y medio ambiente, México.

- (2005), "Short -and Long- run Demand for Energy in Mexico: A Cointegration Approach", Energy Policy, 33 (9), pp. 1179-1185.

Goodwin, P. B. (1992), “A Review of New Demand Elasticities with Special Reference to Short and Long Run Effects on Price Changes", Journal of Transport Economics and Policy, 25 (2), pp. 155-169.

__ , J. Dargay, y M. Hanly (2004), "Elasticities of Road Traffic and Fuel Consumption with Respect to Price and Income: A Review", Transport Reviews, 24 (3), pp. 275-292.

Graham, D. J., y S. Glaister (2002), “The Demand for Automobile Fuel a Survey of Elasticities", Journal of Transport Economics and Policy, 36 (1), pp. 1-26.

(2004), "Road Traffic Demand Elasticity Estimates: A review", Transport Reviews, 24 (3), pp. 261-274.

Granger, C. W. J., y P. Newbold (1974), “Spurious Regressions in Econometrics”, Journal of Econometrics, 2 (2), pp. 306-333.

Hamilton, D. J. (1994), Times Series Analysis, Nueva Jersey, Princeton University Press, Princeton.

Haro, A., y J. L. Ibarrola (2000), "Cálculo de la elasticidad precio de la demanda de gasolina en la zona fronteriza norte de México", Gaceta de Economía, 6 (11), pp. 237-262.

Higgins, P. A. T., y M. Higgins (2005), “A Healthy Reduction in Oil Consumption and Carbon Emissions", Energy Policy, 33 (1), pp. 1-4.

Hodrick, R. J., y E. C. Prescott (1997), "Postwar U.S. Business Cycles: An Empirical Investigation", Journal of Money, Credit and Banking, 29 (1), pp. 1-16.

Hunt, L. C., C. Salgado, y A. Thrope (1999), “The Policy of Power and Power of Policy in Honduras", Journal of Energy and Development, 25 (1), pp. 1-36. 
Instituto Nacional de Ecología (INE) (2006), Inventario Nacional de Emisiones de Gases de Efecto Invernadero (INEGEI): 1990-2002, México.

IPCC (2006), 2006 IPCC Guidelines for National Greenhouse Gas Inventories. Volumen 2 (Energy), preparado por the National Greenhouse Gas Inventories Programme, H. S Eggleston, L. Buendia, K. Miwa, T. Ngara, y K. Tanabe (comps), Japón, Published IGES.

Johansen, S. (1988), "Statistical Analysis of Cointegration Vectors", Journal of Economic Dynamics and Control, 12 (2-3), pp. 231-254.

(1992), "Cointegration in Partial Systems and the Efficiency of Single-Equation Analysis", Journal of Econometrics, 52 (3), pp. 389-402.

(1995), Likelihood-Based Inference in Cointegrated Vector Autoregressive Models, Oxford University Press.

Kayser, H. A. (2000), "Gasoline Demand and Car Choice: Estimating Gasoline Demand Using Household Information”, Energy Economics, 22 (3), pp. 331-348.

Kosugi, T., K. Tokimatsu, y H. Yoshida (2005), "Evaluating New $\mathrm{CO}_{2}$ Reduction Technologies in Japan Up to 2030", Technological Forecasting and Social Change, 72 (7), pp. 779-797.

Kwiatkowsky, D., P. B. C. Phillips, P. Schmidt, y Y. Shin (1992), “Testing the Null Hypothesis of Stationarity Against the Alternative of a Unit Root: How Sure Are We That Economic Time Series Have a Unit Root?", Journal of Econometrics, 54 (1-3), pp. 159-178.

Lenz, H. P., S. Prüller, y D. Gruden (2003), "Means of Transportation and Their Effect on the Environment", en D. Gruden (comp.), The Handbook of Environmental Chemistry, parte T, “Traffic and Environment”, Vol. 3, pp. 107-173.

Litman, T. (2005), "Efficient Vehicles Versus Efficient Transportation: Comparing Transportation Energy Conservation Strategies", Transport Policy, 12 (2), pp. 121129.

Lutsey, N. P., y D. Sperling (2007), “Canada's Voluntary Agreement on Vehicle Greenhouse Gas Emissions: When the Details Matter", Transportation Research Part D: Transport and Environment, 12 (7), pp. 474-487.

MacKinnon, J. G., A. A. Haug, y L. Michelis (1999), "Numerical Distribution Functions of Likelihood Ratio Tests for Cointegration", Journal of Applied Econometrics, 14 (5), pp. 563-577.

Maddala, G. S., y I. Kim (1998), Unit Roots, Cointegration and Structural Change, Cambridge University Press. 
Ministerio de Medio Ambiente y Medio Rural y Marino (MARM) (2010), Inventario de gases de efecto invernadero de España, edición 2006 (serie 1990-2008). Sumario de resultados, Madrid, Subdirección General de Calidad Ambiental.

Martín, V. J., R. J. Llebot, R. E. Padilla, y E. V. Alcántara (2007), Aspectos económicos del cambio climático en España, Caixa Catalunya, monográfico.

Masih, A. M. M., y R. Masih (1997), "On the Temporal Causal Relationship Between Energy Consumption, Real Income, and Prices: Some New Evidence from Asian-Energy Dependent NICS Based on a Multivariate Cointegration/Vector Error-Correction Approach”, Journal of Policy Modeling, 19 (4), pp. 417-440.

Ng, S., y P. Perron P. (1995), "Unit Root Tests in ARMA Models with Data Depend Methods for the Selection of the Truncation Lag", Journal of the American Statistical Association, 90 (429), pp. 68-281.

Nicol, C. J. (2003), "Elasticities of Demand for Gasoline in Canada and the United States", Energy Economics, 25 (2), pp. 201-214.

Oum, T. H. (1989), “Alternative Demand Models and Their Elasticity Estimates”, Journal of Transport Economics and policy, 23 (2), pp. 163-187.

Patterson, K. (2000), An Introduction to Applied Econometrics: A Time Series Approach, St. Martin's Press.

Paz, S. (2008), "El alza de las gasolinas continuará indefinidamente”, Infogas, 66 (10), pp. 62-66.

Pesaran, M. H., y Y. Shin (1999), “An Autoregressive Distributed Lag Modelling Approach to Cointegration Analysis", en Strom Steinar (comp.), Econometrics and Economics Theory in the 20th Century: The Ragnar Frisch Centennial Symposium, Cambrige University Press.

Phillips, P. C. B., y P. Perron (1988), “Testing for Unit Root in Time Series Regression”, Biemetrica, 75 (2), pp. 335-346.

Polemis, M. L. (2006), "Empirical Assessment of the Determinants of Road Energy Demand in Greece", Energy Economics, 28 (3), pp. 385-403.

Ramanathan, R. (1999), "Short- and Long-Run Elasticities of Gasoline Demand in India: An Empirical Analysis Using Cointegration Techniques", Energy Economics, 21 (4), pp. 321-330.

Rao, B. B., y G. Rao (2009), "Cointegration and the Demand for Gasoline”, Energy Policy, 37 (10), pp. 3978-3983.

Reyes, M. O. (2009), "La demanda de gasolinas en México: Efectos y alternativas ante el cambio climático", Documento de Trabajo, Departamento de Economía Aplicada de la Universidad Autónoma de Barcelona. 
Sa'ad, S. (2009a), "An Empirical Analysis of Petroleum Demand for Indonesia: An Application of the Cointegration Approach", Energy Policy, 37 (11), pp. 43914396.

— (2009b), "Transportation Demand for Petroleum Products in Indonesia: a Time Series Analysis", OPEC Energy Review, 33 (2), pp. 140-154.

Samimi, R. (1995), "Road Transport Energy Demand in Australia", Energy Economics, 17 (4), pp. 329-339.

Schafer, A., y D. G. Victor (1999), "Global Passenger Travel: Implications for Carbon Dioxide Emissions", Energy, 24 (8), pp. 657-679.

— y H. D. Jacoby (2006), "Vehicle Technology Under $\mathrm{CO}_{2}$ Constraint: A General Equilibrium Analysis”, Energy Policy, 34 (9), pp. 975-985.

Schipper, L., C. Marie-Lilliu, y L. Fulton (2002) "Diesels in Europe: Analysis of Characteristics, Usage Patterns, Energy Savings and $\mathrm{CO}_{2}$ Emission Implications", Journal of Transport Economics and Policy, 36 (2), pp. 305-340.

Secretaría de Comunicaciones y Transportes (2008), "Programa Sectorial de Comunicaciones y Transporte (2007-2012)", Ciudad de México.

Secretaría de Energía (Sener) (2008), Prospectivas de petrolíferos: 2008-2017, México.

Sperling, D. (2004), Environmental Impacts Due to Urban Transport, en H. Nakamura, Y. Hayashi, y A. D. May (comps.), Urban Transport and the Environment. An International Perspective, World Conference on Transport Research Society, Institute for Transport Policy Studies, Oxford, Elsevier, pp. 99-189.

Stanley, K. J., D. A. Hensher, y C. Loader (2009), "Road Transport and Climate Change: Stepping Off the Greenhouse Gas", Transportation Research Part A: Policy and Practice, doi:10.1016/j.tra.2009.04.005.

Sterner, T. (2007), "Fuel Taxes: An Important Instrument for Climate Policy", Energy Policy, 35 (6), pp. 3194-3202.

_ y C. A. Dahl (1992), "Gasoline Demand Modelling: Theory and Application", en Thomas Sterner (comp.), International Energy Modelling, Londres, Chapman and Hall.

Sullivan, J. L., R. E. Baker, B. A. Boyer, R. H. Hammerle, T. E. Kenney, L. Muniz, y T. J. Wallington (2004), " $\mathrm{CO}_{2}$ Emission Benefit of Diesel (versus gasoline) Powered Vehicles”, Environmental Science and Technology, 38 (12), pp. 3217-3223.

Uri, N. D., y R. Boyd (1999), “A Note on the Economic Impact of Higher Gasoline and Electricity Prices in Mexico", Journal of Policy Modeling, 21 (4), pp. 527-534.

Weiss, M. A., J. B. Heywood, A. Schäfer, y V. K. Natarajan (2003), “A Comparative Assessment of Advanced Fuel Cell Vehicles", MIT Laboratory for Energy and the Environment Report, MIT LFEE 2003-001 RP. 
Wheaton, W. C. (1982), "The Long-Run Structure of Transportation and Gasoline Demand", The Bell Journal of Economics, 13 (2), pp. 439-454.

Wohlgemuth, N. (1997), "World Transport Energy Demand Modeling: Methodology and Elasticities", Energy Policy, 25 (14-15), pp. 1109-1119.

Zachariadis, T., y N. Kouvaritakis (2003), "Long-term Outlook of Energy Use and $\mathrm{CO}_{2}$ Emissions from Transport in Central and Eastern Europe”, Energy Policy, 31(8), pp. 759-773.

Zervas, E., S. Poulopoulos y C. Philippopoulos (2006), " $\mathrm{CO}_{2}$ Emissions Change from the Introduction of Diesel Passenger Cars: Case of Greece", Energy, 31 (14), pp. 2915-2925. 